\title{
FDM computation of seismic wavefield for an axisymmetric earth with a moment tensor point source
}

\author{
Genti Toyokuni and Hiroshi Takenaka \\ Department of Earth and Planetary Sciences, Kyushu University, Hakozaki 6-10-1, Fukuoka 812-8581, Japan
}

(Received April 28, 2006; Revised June 27, 2006; Accepted July 4, 2006; Online published September 15, 2006)

\begin{abstract}
Axisymmetric modeling has been playing an important role in global seismic waveform modeling since it can correctly model geometrical spreading effects in 3-D within computational resources comparable to 2-D modeling. However, in the previous investigations on axisymmetric modeling, seismic sources were restricted to axisymmetric sources such as an explosive source. In this paper, we propose implementation of an arbitrary moment tensor point source to axisymmetric modeling using the finite-difference method (FDM). The validity and efficiency of this technique are demonstrated by comparing synthetic seismograms with analytical solutions for a homogeneous earth model, as well as with the DSM synthetics for a spherically symmetric earth. We also show a numerical example with a subducting slab structure.
\end{abstract}

Key words: Axisymmetric modeling, moment tensor, synthetic seismogram, finite-difference method (FDM).

\section{Introduction}

In order to model seismic wave propagation in realistic structural models with strong lateral heterogeneity, we should adopt numerical procedures such as the finitedifference method (FDM). Synthetic seismogram calculations by these numerical methods, however, for full 3-D situations are still computationally intensive and costly even on parallel hardware. To save on the computational resources, we often employ methods with idealized simple models. One of these methods, the so-called axisymmetric modeling, uses a structure model with rotational symmetry about an axis through a seismic source, and then solves the elastodynamic equation in cylindrical or spherical coordinates. The axisymmetric modeling has been a powerful approach for investigating basic phenomena of seismic waves, developing new techniques, checking a new method or new code, or for finding optimal values of some computational parameters in preparation for more complex modeling (Takenaka et al., 1999). When the geometrical symmetry is presented in a model, by an explicit use of this symmetry, we can reduce computational size in both computation time and memory, because only a subdomain is required for the calculation. The wavefield is then determined by the type of symmetry, instead of adopting the entire structural model.

In the field of global seismology, axisymmetric modeling has performed a particularly important role since it can correctly model the 3-D geometrical spreading effects with computational resources comparable to 2-D modeling. Following the early work by Alterman et al. (1970), many authors have used axisymmetric modeling with the FDM: for acoustic waves (Thomas et al., 2000), for $S H$ waves (Igel and Weber, 1995; Chaljub and Tarantola, 1997; Igel and

Copy right(c) The Society of Geomagnetism and Earth, Planetary and Space Sciences (SGEPSS); The Seismological Society of Japan; The Volcanological Society of Japan; The Geodetic Society of Japan; The Japanese Society for Planetary Sciences; TERRAPUB.
Gudmundsson, 1997), and for $P$-SV waves (Igel and Weber, 1996). Furthermore, Toyokuni et al. (2005) proposed the quasi-spherical approach as an extension of axisymmetric modeling to treat an asymmetric 2-D structure with respect to the source axis. Nevertheless, in conventional axisymmetric modeling, the sources also have to be axisymmetric, which limits the source types to toroidal sources ( $S H$ waves), explosions, or vertical forces ( $P-S V$ waves), though most of earthquakes are caused by double-couple sources. For moment tensor sources, including doublecouple sources, we need to consider the coupling between $P-S V$ and $S H$ waves. In this paper, we provide a way to implement arbitrary moment-tensor point sources to the axisymmetric modeling with the FDM. Although this paper focuses only on a point source represented by symmetric moment tensor, we can treat vector dipoles, CLVDs, explosive sources, and shear dislocation sources, which provide ample description of natural seismic sources (Aki and Richards, 2002). We also consider only an isotropic elastic medium, while the axisymmetric modeling can also be applied to anisotropic or viscoelastic case.

\section{Treatment of Moment Tensor Point Source}

We solve the 3-D elastodynamic equation in spherical coordinates $(r, \theta, \phi)$ for a point source located on the $\theta=0$ axis. For instance, the equation for the vertical component of particle velocity $v_{r}$ is

$$
\begin{aligned}
& \rho \frac{\partial v_{r}}{\partial t}=\partial_{r} \sigma_{r r}+r^{-1} \partial_{\theta} \sigma_{r \theta}+r^{-1} \sin ^{-1} \theta \partial_{\phi} \sigma_{r \phi} \\
& +r^{-1}\left(2 \sigma_{r r}-\sigma_{\theta \theta}-\sigma_{\phi \phi}+\sigma_{r \theta} \cot \theta\right)+f_{r},
\end{aligned}
$$

where $t$ is time, $\rho$ is the density, and $\sigma_{i j}$ are the components of stress tensor. The axisymmetric modeling uses structures which are invariant in $\phi$ direction, so that it considers only a cross section along a great circle of the spherical earth model. 
Table 1. $\theta$-symmetry of field quantities.

\begin{tabular}{c|ccccccc}
\hline Source & $\hat{v}_{r}^{m}$ & $\hat{v}_{\theta}^{m}$ & $\hat{v}_{\phi}^{m}$ & $\hat{\sigma}_{k k}^{m}$ & $\hat{\sigma}_{\theta \phi}^{m}$ & $\hat{\sigma}_{r \theta}^{m}$ & $\hat{\sigma}_{r \phi}^{m}$ \\
\hline (a) & $\mathrm{S}$ & $\mathrm{A}$ & $\mathrm{A}$ & $\mathrm{S}$ & $\mathrm{S}$ & $\mathrm{A}$ & $\mathrm{A}$ \\
(b) & $\mathrm{A}$ & $\mathrm{S}$ & $\mathrm{S}$ & $\mathrm{A}$ & $\mathrm{A}$ & $\mathrm{S}$ & $\mathrm{S}$
\end{tabular}

S: symmetric; A: anti-symmetric with respect to lines $\theta=0$ and $\theta=\pi$. Type (a) contains moment tensor elements with expansion order $m=0$ (with nonzero moment tensor components, $M_{11}=M_{22}$, and $M_{33}$ ), and $m=2\left(M_{11}=-M_{22}\right.$, and $\left.M_{12}=M_{21}\right)$, whereas type (b) contains those with $m=1\left(M_{13}=M_{31}\right.$, and $\left.M_{23}=M_{32}\right)$.

We first take Fourier expansion of all physical components appearing in the elastodynamic equation about $\phi$ direction. For example, the expansion of particle velocity components $v_{i}(i=r, \theta, \phi)$ may be

$$
\begin{aligned}
& v_{i}(t, r, \theta, \phi)=\hat{v}_{i}^{0}(t, r, \theta) \\
& +\sum_{m=1}^{2}\left\{\hat{v}_{i C}^{m}(t, r, \theta) \cos m \phi+\hat{v}_{i S}^{m}(t, r, \theta) \sin m \phi\right\},
\end{aligned}
$$

where $m$ denotes the order of expansion, $\hat{v}_{i}^{0}, \hat{v}_{i C}^{m}$, and $\hat{v}_{i S}^{m}$ $(m=1,2)$ are expansion coefficients. $C$ and $S$ indicate coefficients of cosine and sine terms, respectively. For each component of stress tensor and body forces, we take the same expansion. In this problem, it is sufficient to take the expansion order up to $m=2$ because of the radiation patterns for moment tensor components. This is a similar approach to semi-analytic methods with the vector harmonic function (wavefunction) expansion for the case of a general point source in a 1-D earth model (e.g., Takeuchi and Saito, 1972; Aki and Richards, 2002), and it can also be applied to a non-symmetric moment tensor source as well as to a symmetric moment tensor one we treat here. Substituting the Fourier expansions of the field quantities such as Eq. (2) into the elastodynamic equation (Eq. (1)) and rearranging the results as for the constant terms, $\cos m \phi$ terms, and $\sin m \phi$ terms give five closed systems of partial differential equations (PDEs) about expansion coefficients. Through this process, an arbitrary moment-tensor point source is decomposed into five moment-tensor elements-i.e., the element for axisymmetric source $(m=0)$ and the elements corresponding to four shear dislocation sources (two types of pure vertical dip-slip sources, nonzero components of $M_{13}=M_{31}$, and those of $M_{23}=M_{32}$; two types of pure strike-slip sources, $M_{11}=-M_{22}$, and $\left.M_{12}=M_{21}\right)$. For implementation, we further divide the axisymmetric source into two elements: the vertical component $M_{33}$ and the horizontal $M_{11}=M_{22}$, where the other components of moment tensor are zero for each case. Note that calculations for any combinations of these axisymmetric elements can be performed by one computation changing the weight for each element. We can model seismic wave propagation due to an arbitrary moment tensor point source by solving these equation systems about expansion coefficients using a numerical method such as the FDM, and then substituting them into Eq. (2).

\section{FDM Implementation}

In order to solve the five systems of PDEs of expansion coefficients, we adopt a velocity-stress staggered-grid finite-difference scheme. The staggered-grid system is the

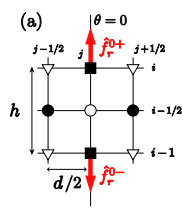

(d)
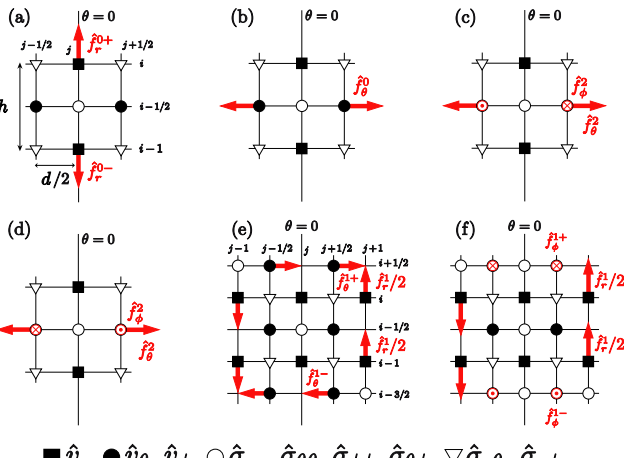

Fig. 1. FDM implementation of Cartesian moment tensor elements to the FDM scheme using equivalent body-force components $\hat{f}_{r}, \hat{f}_{\theta}$, and $\hat{f}_{\phi}$ ( $h=\Delta r, d=r_{s} \Delta \theta$ ): (a) $M_{33}$; (b) $M_{11}=M_{22}$; (c) $M_{11}=-M_{22}$; (d) $M_{12}=M_{21}$; (e) $M_{13}=M_{31}$; (f) $M_{23}=M_{32}$.

same as that described in Igel and Weber (1996) although we chose the grids for $\hat{v}_{\phi}, \hat{\sigma}_{\theta \phi}$, and $\hat{\sigma}_{r \phi}$ to be the same position as those for $\hat{v}_{\theta}, \hat{\sigma}_{k k}$ (normal stress components), and $\hat{\sigma}_{r \theta}$, respectively. The grids for $\hat{v}_{r}, \hat{\sigma}_{k k}$, and $\hat{\sigma}_{\theta \phi}$ lie on the source axis $\theta=0$.

In the axisymmetric modeling, we perform the FDM calculation on a computational domain with an angular range of $\theta \geq 0$. However, to calculate $\theta$-derivatives $\partial / \partial \theta$ near and on the axis $\theta=0$ (and $\theta=\pi$ ), we need values of field quantities on grid points at $\theta<0$ near the axis. When we assign the $\theta \geq 0$ domain on a half plane of a cross section of the earth at $\phi=\phi_{0}$, the $\theta<0$ domain corresponds to another half at $\phi=\phi_{0}+\pi$. Therefore, the field quantities have symmetric or anti-symmetric properties with respect to the $\theta=0$ and $\theta=\pi$ axes due to each of the six moment tensor elements, based on symmetry of radiation patterns of wavefields excited by these elements. For the element corresponding to a pure strike-slip source $M_{12}=M_{21}=M_{0}$, as an example, $\hat{v}_{r}$ is symmetric around line $\theta=0$, whereas the angular $(\theta)$ and transverse $(\phi)$ component of particle velocity, $\hat{v}_{\theta}$ and $\hat{v}_{\phi}$, are anti-symmetry, i.e.,

$$
\begin{gathered}
\hat{v}_{r}^{2}(\cdot,-\theta)=\hat{v}_{r}^{2}(\cdot, \theta), \\
\hat{v}_{\theta}^{2}(\cdot,-\theta)=-\hat{v}_{\theta}^{2}(\cdot, \theta), \quad \hat{v}_{\phi}^{2}(\cdot,-\theta)=-\hat{v}_{\phi}^{2}(\cdot, \theta),
\end{gathered}
$$

where the base vector of each component has been assumed to be continuous across the axis $\theta=0$; e.g., $\boldsymbol{e}_{\phi}(\cdot, \theta=$ $0-)=\boldsymbol{e}_{\phi}(\cdot, \theta=0+)$ for the unit base vector of $\phi, \boldsymbol{e}_{\phi}$.

The symmetric or anti-symmetric properties of the stress components are then derived from Eq. (3), and symmetric property of the structural model through the stressdisplacement relations appear in the elastodynamic equation (e.g., Takenaka et al., 1999). Table 1 shows properties of symmetry or anti-symmetry about the source axis of field quantities radiated by six moment tensor elements. From these relations, we can obtain field quantities at $\theta<0$ from corresponding values at $\theta>0$, so that the calculation of $\theta$-derivatives around the source axis becomes possible.

Now we implement the moment tensor sources on the FD scheme. Figure 1 shows the implementation of six moment tensor elements with body forces. Note that all elements have the same center on a grid for normal stress components. Coefficients corresponding to the body forces 
around a grid cell are set as follows:

(a) $\hat{f}_{r}^{0+}=-\hat{f}_{r}^{0-}=M_{0} / V \Delta r$,

(b) $\hat{f}_{\theta}^{0}=M_{0} / V r_{s} \Delta \theta$,

(c) $\hat{f}_{\theta}^{2}=\hat{f}_{\phi}^{2}=4 M_{0} / V r_{s} \Delta \theta$,

(d) $\hat{f}_{\theta}^{2}=-\hat{f}_{\phi}^{2}=4 M_{0} / V r_{s} \Delta \theta$,

(e) $\hat{f}_{r}^{1}=2 M_{0} / V r_{s} \Delta \theta, \quad \hat{f}_{\theta}^{1+}=-\hat{f}_{\theta}^{1-}=2 M_{0} / V \Delta r$

(f) $\hat{f}_{r}^{1}=2 M_{0} / V r_{s} \Delta \theta, \quad \hat{f}_{\phi}^{1+}=-\hat{f}_{\phi}^{1-}=2 M_{0} / V \Delta r$

where $r_{s}$ is the radius at the source, and $\Delta r$ and $\Delta \theta$ denote the grid spacings in the $r$ and $\theta$ directions, respectively. $V=2 \pi\left(r_{s} \Delta \theta\right)^{2} \Delta r$ for cases (e) and (f), while for the other cases $V=\pi\left(r_{s} \Delta \theta\right)^{2} \Delta r / 4$. Although we explicitly give body forces only for right half of the grid cell $(0 \leq \theta \leq \pi)$, forces on the opposite side $(-\pi<\theta<0)$ are automatically settled by the symmetry in Table 1 .

We use the FDM scheme with second-order accuracy in time and fourth-order accuracy in space. For keeping the fourth-order FD approximation to the terms without spatial derivatives, we use the same identities as in Toyokuni et al. (2005) followed by discretization. We use two different identities, such as

$$
\begin{gathered}
\frac{\hat{\sigma}_{i j}}{\sin \theta}=\frac{1}{\cos \theta} \frac{\partial \hat{\sigma}_{i j}}{\partial \theta}-\tan \theta \frac{\partial}{\partial \theta}\left(\frac{\hat{\sigma}_{i j}}{\sin \theta}\right), \\
\frac{\hat{\sigma}_{i j}}{\sin \theta}=\frac{1}{\sin \theta}\left\{\frac{\partial}{\partial \theta}\left(\theta \hat{\sigma}_{i j}\right)-\theta \frac{\partial \hat{\sigma}_{i j}}{\partial \theta}\right\},
\end{gathered}
$$

$(i, j)=(r, \phi),(\theta, \phi),(\phi, \phi)$, to avoid zero divisions on and near the axes $\theta=0, \pi / 2, \pi$, and $3 \pi / 2$. Furthermore, on the $\theta=0$ and $\theta=\pi$ axes, we exploit the formulae derived from limiting operations with the L'Hospital rule to overcome the singularity on these axes (e.g., Alterman et al., 1970; Takenaka et al., 2003). Through this paper, we adopt a nonuniform grid configuration (Pitarka, 1999) in the vertical coordinate, whereas a uniform grid spacing is used in the angular direction.

\section{Comparison with Analytical Solutions}

Here we demonstrate the validity of the FDM scheme for moment tensor sources described in the previous section, through computations for simple models. We first calculate synthetic seismograms radiated by each moment tensor element (Fig. 1) for a homogeneous earth model with the seismic velocity $v_{p}=8.02 \mathrm{~km} / \mathrm{s}, v_{s}=4.44 \mathrm{~km} / \mathrm{s}$, and density
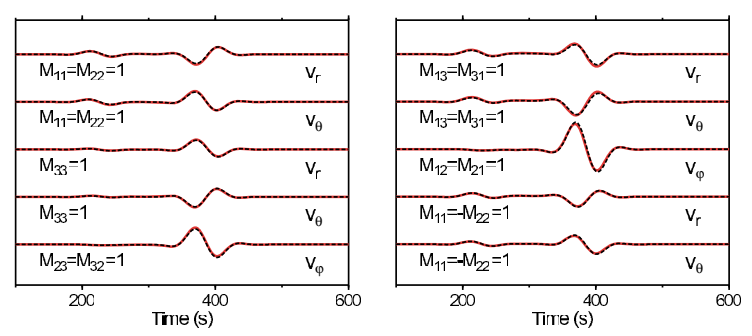

Fig. 2. Synthetic seismograms at an epicentral distance $\Delta=18^{\circ}\left(\phi=0^{\circ}\right)$ for a homogeneous earth model with each of the six moment tensor elements shown in Fig. 1. The FDM results are displayed by solid lines, while the analytical solutions are shown by dashed lines. A low-pass filter $(<1 / 60 \mathrm{~Hz})$ has been applied.

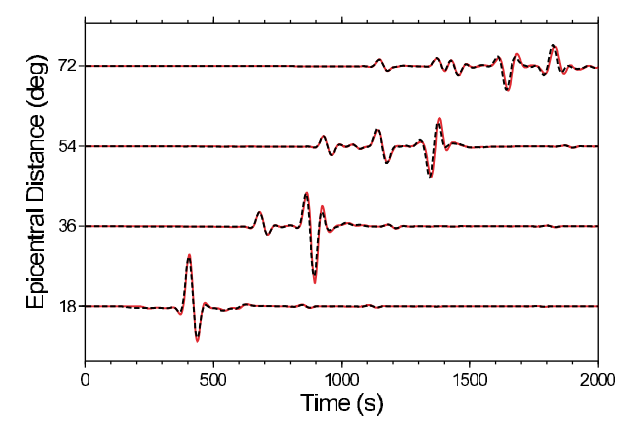

Fig. 3. Synthetic seismograms for the IASP91 model with a moment tensor element $M_{12}=M_{21}$. The transverse component of particle velocity is shown for the FDM (solid lines) and the DSM (dashed lines). The observation points are located at the earth's surface of plane $\phi=0$. A low-pass filter $(<1 / 60 \mathrm{~Hz})$ has been applied.

$\rho=3.36 \mathrm{~g} / \mathrm{cm}^{3}$. The results are compared with the analytical solutions. The model is defined on a $678(r) \times 1800(\theta)$ grid with an angular range of $180^{\circ}$ and a maximum depth of $5321 \mathrm{~km}$. The time increment in the FDM is $0.05 \mathrm{~s}$. We put a 2942-km-depth point source with a source time function as a phaseless bell-shaped pulse with a width of $60 \mathrm{~s}$. In order to keep out reflected waves from the free surface and the other artificial boundary, we put the observation points at a depth of $1942 \mathrm{~km}$. Figure 2 shows the synthetic seismograms at an epicentral distance $\Delta=18^{\circ}\left(\phi=0^{\circ}\right)$ for both the FDM and the analytical solutions. The $v_{r}$ and $v_{\theta}$ components of particle velocity are displayed for cases (a), (b), (c), and (e) in Fig. 1, while the $v_{\phi}$ component is shown for (d) and (f). There is a very good agreement in the travel times, amplitudes, and waveforms for all traces, suggesting that our FDM scheme with moment tensor elements has sufficient accuracy.

Next, we employ the spherically symmetric earth model IASP91 (Kennett and Engdahl, 1991) with a moment tensor element $M_{12}=M_{21}=1$, and compare the results with those obtained by the direct solution method (DSM) (Takeuchi et al., 1996). The DSM can give exact waveforms for spherically symmetric media. We put a $638-\mathrm{km}$-depth seismic source and compare the waveforms on the free surface. The grid configuration, numerical scheme, and source time function are the same as those used in the homogeneous cases. Figure 3 shows the transverse component (i.e. $\phi$-component) of the synthetic seismograms at four epicentral distances $\left(\Delta=18^{\circ}, 36^{\circ}, 54^{\circ}, 72^{\circ}\right)$ for both the axisymmetric FDM and the DSM. There is also a very good agreement among the waveforms for all phases, although there are small travel time differences on latter phases due to the slight differences of discretized structural models between the FDM and DSM schemes.

\section{Numerical Example}

We now illustrate the application of our scheme with a numerical example for a more complex structure. We adopt a model with subducting slab structure stagnating above the 660-km discontinuity superimposed on the IASP91 model. We calculate synthetic seismograms for a 631-km-deep point source with a sub-horizontal fault plane $\left(\phi_{s}=311^{\circ}\right.$, $\left.\delta=11^{\circ}, \lambda=-51^{\circ}\right)$ referring to the 1994 deep Bolivia earthquake $\left(M_{\mathrm{W}}=8.2\right.$; Lundgren and Giardini, 1995). The 


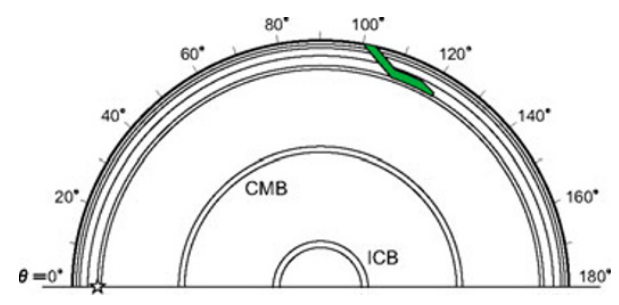

Fig. 4. Model with a slab for numerical example. The stagnant slab is lying on the $660-\mathrm{km}$ discontinuity, with a $+10 \%$ perturbation for the $P$-wave velocity, $S$-wave velocity, and density from the IASP91 model. The star indicates the location of source.

slab structure which is located $\Delta=100^{\circ}$ away from the source axis, as shown in Fig. 4 , is then a rough image of the slab below the Tonga arc (Fukao et al., 2001). The perturbation for the compressional wave velocity, shear wave velocity, and density in the slab are set at $+10.0 \%$ above the IASP91 basis. The grid configuration, numerical scheme, and source time function are also the same as in the previous calculations.

The vertical component of the synthetics at the earth's surface is shown in Fig. 5(a). Differential seismograms of vertical $(r)$ and transverse $(\phi)$ components, calculated by subtraction of the results for the IASP91 from those for the stagnant slab, are displayed in Figs. 5(b) and 5(c), respectively. Most of the differences occur from $\Delta=100^{\circ}$ where the perturbation begins. We can see Rayleigh waves converted from $S P$ and SSSP at the joint of the slab, while Love waves appear due to a similar conversion from $S S$ and SSS.

\section{Discussion and Conclusion}

In this paper, we have proposed a method to implement an arbitrary moment tensor point source into the FDM scheme for axisymmetric modeling with spherical coordinates. We can calculate seismic wave propagation excited by any moment tensor point sources on a 2-D slice of whole axisymmetric earth by performing the computation only in the half domain of the model. Since most earthquakes are caused by double-couple sources, this method is expected to
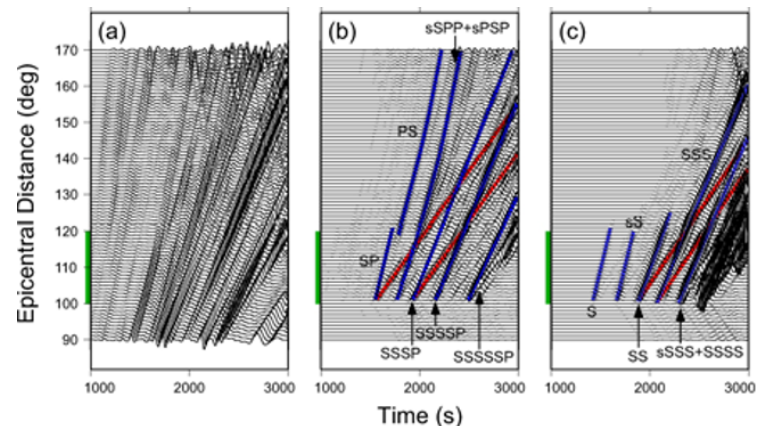

Fig. 5. Synthetic seismograms at the earth's surface $\left(\phi=180^{\circ}\right)$ for the slab model (Fig. 4). (a) Vertical component of the original seismograms (particle velocity). (b) Differential seismograms of $r$-component and (c) $\phi$-component of particle velocity, which indicate the anomalies in travel times and amplitudes caused by the slab. Green bands indicate the angular range of the slab model. Phases affected by the slab are displayed (blue lines) as well as surface waves generated at the root of the slab model (red lines). All traces are shown in the same scale. greatly facilitate the application of the axisymmetric modeling. Although this paper has treated a point source located on $\theta=0$ axis, our method can also treat a source of finite extent. In this case, we may need to add the results of plural simulations for several point sources. For example, the finite source in a subducting slab may require the sum of many wavefields resulting from several point sources to represent a finite source.

Acknowledgments. We would like to thank Dr. Y. Wang for providing us with the DSM synthetic seismograms. Discussion with Prof. B. L. N. Kennett was helpful for this work. The comments of Prof. K. Yomogida and Dr. N. Takeuchi and one anonymous reviewer were helpful for improving our manuscript. Some of figures were created using the Generic Mapping Tools (GMT) software (Wessel and Smith, 1995). This study was partially supported by JSPS.KAKENHI (16540389).

\section{References}

Aki, K. and P. G. Richards, Quantitative Seismology, 2nd ed., 700 pp, University Science Books, Sausalito, CA, 2002.

Alterman, Z. S., J. Aboudi, and F. C. Karal, Pulse propagation in a laterally heterogeneous solid elastic sphere, Geophys. J. R. astr. Soc., 21, 243260, 1970.

Chaljub, E. and A. Tarantola, Sensitivity of $S S$ precursors to topography on the upper-mantle 660-km discontinuity, Geophys. Res. Lett., 24(21), 2613-2616, 1997.

Fukao, Y., S. Widiyantoro, and M. Obayashi, Stagnant slabs in the upper and lower mantle transition region, Rev. Geophys., 39(3), 291-323, 2001.

Igel, H. and O. Gudmundsson, Frequency-dependent effects on travel times and waveforms of long-period $S$ and SS waves, Phys. Earth Planet. Int., 104, 229-246, 1997.

Igel, $\mathrm{H}$. and $\mathrm{M}$. Weber, $\mathrm{SH}$-wave propagation in the whole mantle using high-order finite differences, Geophys. Res. Lett., 22(6), 731-734, 1995.

Igel, $\mathrm{H}$. and M. Weber, P-SV wave propagation in the Earth's mantle using finite differences: Application to heterogeneous lowermost mantle structure, Geophys. Res. Lett., 23(5), 415-418, 1996.

Kennett, B. L. N. and E. R. Engdahl, Traveltimes for global earthquake location and phase identification, Geophys. J. Int., 105, 429-465, 1991.

Lundgren, P. and D. Giardini, The June 9 Bolivia and March 9 Fiji deep earthquakes of 1994: I. Source processes, Geophys. Res. Lett., 22(16), 2241-2244, 1995.

Pitarka, A., 3D elastic finite-difference modeling of seismic motion using staggered grids with nonuniform spacing, Bull. Seism. Soc. Am., 89(1), 54-68, 1999.

Takenaka, H., Y. Wang, and T. Furumura, An efficient approach of the pseudospectral method for modelling of geometrically symmetric seismic wavefield, Earth Planets Space, 51, 73-79, 1999.

Takenaka, H., H. Tanaka, T. Okamoto, and B. L. N. Kennett, Quasicylindrical 2.5D wave modeling for large-scale seismic surveys, Geophys. Res. Lett., 30(21), 2086, doi:10.1029/2003GL018068, 2003.

Takeuchi, H. and M. Saito, Seismic surface waves, Meth. comput. Phys., 11, 217-295, 1972.

Takeuchi, N., R. J. Geller, and P. R. Cummins, Highly accurate P-SV complete synthetic seismograms using modified DSM operators, Geophys. Res. Lett., 23(10), 1175-1178, 1996.

Thomas, Ch., H. Igel, M. Weber, and F. Scherbaum, Acoustic simulation of $P$-wave propagation in a heterogeneous spherical earth: numerical method and application to precursor waves to PKPdf, Geophys. J. Int., 141, 307-320, 2000.

Toyokuni, G., H. Takenaka, Y. Wang, and B. L. N. Kennett, Quasispherical approach for seismic wave modeling in a 2-D slice of a global Earth model with lateral heterogeneity, Geophys. Res. Lett., 32, L09305, doi:10.1029/2004GL022180, 2005.

Wessel, P. and W. H. F. Smith, New version of the generic mapping tools released, EOS Trans. AGU Electron. Suppl., Aug. 15, 1995.

G. Toyokuni (e-mail: toyokuni@geo.kyushu-u.ac.jp) and H. Takenaka (e-mail: takenaka@geo.kyushu-u.ac.jp) 